\title{
Wie sind Rentabilität und Entwicklung zu vereinen? Öffentlich-private Partnerschaften aus der Sicht einer Nichtregierungsorganisation
}

\section{Christine Eberlein}

\section{OpenEdition}

Electronic version

URL: http://journals.openedition.org/sjep/482

DOI: $10.4000 /$ sjep.482

ISSN: 1663-9677

Publisher

Institut de hautes études internationales et du développement

Printed version

Date of publication: 1 octobre 2005

Number of pages: $145-164$

ISBN: 2-88247-059-2

ISSN: 1660-5926

\section{Electronic reference}

Christine Eberlein, « Wie sind Rentabilität und Entwicklung zu vereinen ? Öffentlich-private

Partnerschaften aus der Sicht einer Nichtregierungsorganisation », Schweizerisches Jahrbuch für Entwicklungspolitik [Online], 24-2 | 2005, Online erschienen am: 08 Juni 2010, abgerufen am 08

September 2020. URL : http://journals.openedition.org/sjep/482 ; DOI : https://doi.org/10.4000/sjep. 482

(c) The Graduate Institute 


\title{
Wie sind Rentabilität und Entwicklung zu vereinen? Öffentlich-private Partnerschaften aus der Sicht einer Nichtregierungsorganisation
}

\author{
Christine Eberlein*
}

\section{Einleitung}

Die Aufgaben, welche Regierungen von Entwicklungsländern zu bewältigen haben, sind immens. Dazu gehören der Aufbau der Wirtschaft und der Märkte, der Wasser-, Strom- und Transportinfrastruktur, der Bildungseinrichtungen sowie der Kampf gegen Hunger, Krankheit und Armut. In den meisten Ländern Afrikas haben weniger als 5 Prozent der Menschen Zugang zu Strom. In den schnell wachsenden Städten im Süden lebt die Hälfte der Bevölkerung am Existenzminimum. Nach Schätzungen der UNO haben 1,1 Milliarden Menschen weltweit keinen Zugang zu Trinkwasser und 2,4 Milliarden Menschen sind nicht an Abwasser- und Toilettensysteme angeschlossen ${ }^{1}$.

$\mathrm{Zu}$ Beginn des 21. Jahrhunderts ist den Banken des Nordens und auch der Entwicklungszusammenarbeit die Illusion vergangen, mit auslandsfinanzierten Krediten eine rasche wirtschaftliche Entwicklung armer Länder einleiten zu können. Als Folge der hohen Kreditaufnahmen stecken die Entwicklungsländer seit den 80er Jahren in einer unüberwindbaren Schuldenkrise. Es mangelt an Geldern, weil die armen Länder von der weltwirtschaftlichen Dynamik abgekoppelt sind, und ausländische Direktinvestitionen sowie die internationalen Finanzströme diese Länder umgehen. Da reiche Menschen im Süden ihre Gelder lieber auf Banken im Norden anlegen, werden zu wenig Steuern generiert. Zudem nehmen auch die bilateralen Entwicklungshilfegelder tendenziell ab. Eine wichtige entwicklungspolitische Frage des 21. Jahrhunderts lautet deshalb: Wer soll die öffentlichen Infrastrukturaufgaben wie den Bau von Wasser- und Abwasserleitungen, die Stromversorgung, die Telekommunikation und Spitäler finanzieren, bauen und betreiben? Und wie können Menschen, die von einem Dollar pro Tag leben, kostengünstigen Zugang zu diesen Dienstleistungen erhalten?

Angesichts der knappen Gelder von Regierungen des Südens propagieren internationale Finanzinstitutionen und Geber, wie das Staatssekretariat für Wirtschaft (seco) und die Direktion für Entwicklung und Zusammenarbeit (DEZA) in der Schweiz, dass diese Aufgaben im Rahmen von so genannten „Public-Private Partnerships" (PPPs) in einer Partnerschaft mit dem Privatsektor angegangen werden müssen. Der Grundsatz dieser öffentlich-privaten Partnerschaften lautet: Regierungen sollen nur noch öffentliche Aufgaben definieren und regulieren,

* Leiterin des Programms „Internationale Finanzbeziehungen“, Erklärung von Bern.

1 UN-Millennium Project, Jeffrey D. Sachs (dir.): „Investing in Development: A Practical Plan to Achieve the Millennium Development Goals“, UNDP, 2005, Chap. 1. 
während die Privatwirtschaft den operativen Teil übernimmt. In der Theorie mag dies rosig erscheinen. Aber funktioniert das angebliche Erfolgsmodell auch in der Praxis? Als Nichtregierungsorganisation beleuchten wir in diesem Artikel insbesondere öffentlich-private Partnerschaften im Bereich der Basisinfrastruktur sowie aus der Sicht von am Existenzminimum lebenden Menschen, die sich fragen, ob sie sich die Grundversorgung noch leisten können, wenn sie von privaten Unternehmen angeboten wird. Wir beschränken uns dabei auf PPPs in lebensnotwendigen Bereichen der öffentlichen Versorgung, wie zum Beispiel Wasserversorgung, Abwasserentsorgung und Stromversorgung sowie der Bau von Häusern, Spitälern und Transportsystemen. Im Unterschied zu PPPs in den Bereichen Bildung oder Telekommunikation geht es dabei um grundlegende Leistungen, die praktisch per Gesellschaftsvertrag als Staatsaufgabe definiert sind.

Es gibt im Norden durchaus Bereiche, in denen öffentlich-private Partnerschaften Erfolg haben. Ein Beispiel ist die Müllabfuhr von Gemeinden. Gemeinderäte garantieren die Abholung der Abfälle, und private Unternehmen besorgen sie pünktlich. Bei Beschwerden wechselt die Gemeinde einfach das Unternehmen. Auch öffentlich-private Partnerschaften im Telekommunikationssektor funktionieren gut, weil die Einstiegskosten hier niedrig sind und genügend Anbieter im Wettbewerb stehen. Hingegen zeigt das bekannte Beispiel der britischen Bahn „Railtrack“, dass PPPs durchaus Probleme mit sich bringen können und am Ende wieder den öffentlichen Haushalt belasten: Die britische Regierung hatte aus Geldmangel im Rahmen einer Public-Private Partnership Bahnlinien, Gleise und Signale privatisiert. Als der private Vertragspartner Railtrack in finanzielle Schwierigkeiten geriet, wurde er mit Steuergeldern gerettet. Mittlerweile werfen zahlreiche Regierungspartnerschaften mit privat geleiteten Spitälern, Schulen, Gefängnissen, Transportsystemen sowie Wasser- und Stromversorgungen weltweit diverse Probleme auf. Diesen Bereichen ist gemeinsam, dass dort hohe Investitionskosten und langfristige Verträge mit den privaten Partnern erforderlich sind und die privaten Anbieter dadurch eine Monopolstellung haben. Arme Bevölkerungsschichten beklagen sich vor allem über unbezahlbar gestiegene Kosten. Auf den Philippinen, in Südafrika, Indien, Ghana und Bolivien beispielsweise wehrten sich Betroffene in tagelangen Demonstrationen gegen die massiv gestiegenen Wasserpreise. Die Regierungen sahen sich schliesslich gezwungen, die PPP-Verträge zu kündigen.

Die Frage, wer die öffentlichen Dienstleistungen im Infrastrukturbereich übernehmen und kontrollieren soll, ist deshalb so schwierig, weil es gilt, unterschiedliche Interessengruppen, von Regierungen über Unternehmen $\mathrm{zu}$ verschiedenen Bevölkerungsschichten, gerecht $\mathrm{zu}$ werden. Tatsache ist, dass Regierungsbeamte, Politiker und Konzerne allzu oft weiterhin der Illusion nachhängen, sie könnten Pläne machen und diese umsetzen, ohne die Anliegen der Nutzniesser einzubeziehen. Wir argumentieren, dass sich Entscheidungsträger den Bedürfnissen der betroffenen Interessengruppen stellen müssen, da sonst eine vermeintliche Lösung mehrere neue Probleme aufwerfen kann. In diesem Sinne behaupten wir, dass das Modell der PPPs nur erfolgreich sein kann, wenn es als offener Prozess verstanden wird, der die Interessen aller Akteure gleichberechtigt einbezieht.

Im ersten Kapitel des Artikels zeigen wir nach einer Abgrenzung des Begriffs „Public-Private Partnerships“, welche Hoffnungen und Befürchtungen die verschiedenen Interessengruppen mit den Partnerschaften verbinden. 
Im zweiten Kapitel wollen wir mit einem kurzen, historischen Rückblick die politische Entstehungsgeschichte von PPPs verständlicher machen. Wir argumentieren, dass öffentlich-private Partnerschaften das jüngste Produkt der Politik der internationalen Finanzinstitutionen sind, die nun seit sechzig Jahren eine Kette von Instrumenten zur Entwicklungsfinanzierung propagieren, ohne vorher deren Aus- und Wechselwirkungen hinreichend untersucht zu haben.

Im dritten Kapitel analysieren wir zum einen, ob Regierungen in Entwicklungsländern überhaupt dafür gerüstet sind, PPPs zu managen. Zum anderen beleuchten wir anhand von Fallbeispielen Spannungsfelder der Partnerschaft zwischen privaten Unternehmen und öffentlichen Institutionen sowie mögliche negative Folgen für die arme Bevölkerung.

Im vierten Kapitel befassen wir uns mit der teilprivatisierten Wasserversorgung von Armenvierteln in El Alto, oberhalb der bolivianischen Hauptstadt La Paz. Das Projekt ist aus verschiedenen Gründen interessant: Zum einen sollte es mit der Unterstützung des Staatssekretariats für Wirtschaft (seco) ein Modell für öffentlich-private Partnerschaften im Bereich der Wasserversorgung in armen urbanen Zentren werden. Zum anderen sorgte das Projekt im Dezember 2004 für internationale Schlagzeilen, als die Bevölkerung von El Alto auf die Strasse ging und gegen die Privatisierung protestierte. Die bolivianische Regierung löste den Konzessionsvertrag mit dem privaten Partner schliesslich auf. Am Beispiel der Kooperative SAGUAPAC, welche die Wasserversorgung der bolivianischen Millionenstadt Santa Cruz hervorragend verwaltet, zeigen wir, dass es durchaus Alternativen zu PPPs gibt.

Abschliessend ziehen wir Schlussfolgerungen und geben Empfehlungen für zukünftige PPPs.

\section{Public-Private-Partnerships (PPPs)}

\section{Entstehung und Abgrenzung des Begriffs „Public-Private-Partnerships"}

Der Begriff der öffentlich-privaten Partnerschaften umfasst eine Vielzahl unterschiedlicher Beziehungen zwischen öffentlichen Institutionen (wie Regierung und Gemeinden) und privaten Unternehmen, um Projekte, die vorher Aufgabe des öffentlichen Dienstes waren, zu planen oder durchzuführen. Osbourne und Gabler $^{2}$ (1992) beschreiben PPPs als „das Rudern und Steuern eines Bootes“. Der Staat steuert, während die privaten Akteure das Boot rudern, um öffentliche Dienstleistungen und Infrastruktur bereitzustellen. Die Zusammenarbeit zwischen Staat und Privatsektor ist dabei vertraglich geregelt. Die Leistungen der Partner sollen sich theoretisch ergänzen, so dass beide zusammen ein Ziel effizienter erreichen können. Das Risiko soll, wenn möglich, geteilt oder von dem am besten geeigneten Partner getragen werden. Es stellt sich immer wieder die Frage, ob das Auslagern von öffentlichen Aufgaben auch unter PPPs fällt. Wichtig ist in dieser Hinsicht die Unterscheidung, dass bei öffentlich-privaten Partnerschaften nicht der Staat den privaten Leistungsauftrag bezahlt und kontrolliert, sondern direkt der Empfänger, also der Kunde. Dadurch steigt das Risiko für das private Unternehmen.

2 S. Osbourne, T. Gabler, Reinventing Government: How the Entrepreneurial Spirit is Transforming the Public Sector, Reading (Massachusetts), Addison-Wesley Pub. Co., 1992. 
Als erste multilaterale Institution propagierte die Weltbank zu Beginn der 90er Jahre PPPs im Infrastrukturbereich ${ }^{3}$. Seit Mitte der 90er Jahre fördern auch bilaterale Entwicklungsagenturen zunehmend so genannte „Entwicklungspartnerschaften“. 1999 folgte die UNO mit dem Global Compact, um globale Konzerne in die Umsetzung der UN-Politik im Bereich sozialer und wirtschaftlicher Rechte in Partnerschaften einzubinden. Allerdings handelt es sich dabei um weitgehend unverbindliche und kaum überprüfbare Selbstverpflichtungen der Wirtschaft. Auch an der UN-Konferenz über Entwicklungsfinanzierung, die 2002 in Monterrey (Mexiko) stattfand, betonten Regierungen die steigende Bedeutung von PPPs, um angesichts knapper Entwicklungshilfegelder überhaupt noch die notwendigen Gelder für die Erreichung der Millenniums-Entwicklungsziele 4 aufzubringen.

Die internationalen Finanzgeber unterscheiden öffentlich-private Partnerschaften von der Privatisierung, weil es sich bei letzterer um den definitiven Verkauf ehemals staatlicher Institutionen handelt. Wir argumentieren dagegen, dass der praktische Unterschied gering ist. Beide Instrumente beruhen auf juristischen Verträgen und bedürfen starker Überwachung, transparenter Rechtsprechung und stetiger öffentlicher Kontrolle, damit der private Partner die öffentliche Aufgabe wahrnimmt. Internationale Studien über PPPs zeigen zudem, dass vor allem Partnerschaften im Rahmen von Dezentralisierungsstrategien von den Interessen des Privatsektors dominiert werden und daher eine Form von Privatisierung sind ${ }^{5}$.

\section{Die Interessen der verschiedenen Akteure}

Wie bereits angesprochen, verbinden die verschiedenen Interessengruppen mit PPPs unterschiedliche Hoffnungen, aber auch Befürchtungen:

Regierungen erhoffen sich von der Auslagerung öffentlicher Aufgaben neben dem Ersparniseffekt und der Verbesserung von Effizienz und Qualität der Dienstleistungen unter anderem technische Expertise, Innovationen und besseres Risikomanagement. Im Vergleich zu Regierungen könnten private Unternehmen eine grössere Nähe zwischen Kunden und Anbietern garantieren, um entsprechend schneller und besser auf Kundenwünsche oder Marktänderungen einzugehen. Südliche Regierungen versuchen, ausländische Investoren mit Steuervergünstigungen, Garantien und staatlicher Unterstützung anzulocken, um deren Risiken zu minimieren.

Die Privatwirtschaft steht der Förderung von PPPs durchaus aufgeschlossen gegenüber. Im Vordergrund steht die Aussicht, neue Märkte zu erobern und Gewinne zu erhöhen. Partnerschaften mit dem Staat können für die Unternehmen möglicherweise rechtliche und politische Hürden verringern und Kosten durch Auslagerung von Ausbildungskosten senken.

\footnotetext{
World Bank, World Development Report 1994, Infrastructure for Development, Washington, D.C. Die UN-Millenniumsziele fordern die Halbierung der weltweiten Armut bis zum Jahr 2015.

F. Miraftab, „Public-Private Partnerships, the Trojan Horse of Neoliberal Development ?“, Journal of Planning Education and Research, Vol. 24, No 1, 2004, S. 89-101; T. Kessler, Putting the Private Sector in its Place, part 1, „Issues and Evidence in the Reform of Water and Electricity Services“, Takoma Park, USA, Citizens' Network on Essential Services, 2002.
} 
Die internationale Entwicklungszusammenarbeit propagiert PPPs in der Hoffnung, dadurch den Rückgang öffentlicher Entwicklungshilfegelder kompensieren zu können. Die Synergieeffekte mit der Wirtschaft sollen Regierungen entlasten und „unrentable Projektruinen“ verhindern. Dabei geht es durchaus auch um die Export- und Wirtschaftsförderung von Unternehmen des Nordens bei der Erschliessung neuer Märkte in Entwicklungsländern.

„Öffentlich-private Partnerschaften sind in der Lage, Infrastruktur und andere Dienstleistungen bereitzustellen, ohne die Finanzen eines Landes zu überlasten", schreibt der Internationale Währungsfonds (IWF) in seiner Publikation Finance \& Development im Dezember 20046. Dahinter steht die Vorstellung, Einfluss auf die Privatwirtschaft nehmen zu können und dabei die Investitionen in entwicklungspolitisch wünschenswerte Bahnen zu lenken. Gleichzeitig warnen die Autoren, dass PPPs Grenzen haben und nur funktionieren, wenn Voraussetzungen einer demokratischen Kontrolle gegeben $\operatorname{sind}^{7}$.

Die lokale Zivilgesellschaft ist in erster Linie daran interessiert, Zugang zu Basisdiensten zu bekommen, die sie sich leisten kann, ohne dafür auf andere lebensnotwendige Ausgaben verzichten zu müssen. Ebenso wichtig ist, dass die Versorgung langfristig effizient funktioniert. Die Zivilgesellschaft im Süden hat traditionell mehr Vertrauen zu ihren Regierungen als zu ausländischen Privatunternehmen. Daher wünschen sich die meisten eher eine Versorgung durch den Staat als durch Unternehmen. Widerstand herrscht vor allem gegenüber Massnahmen, die von internationalen Finanzgebern von aussen vorgegeben werden, ohne auf die in jedem Land unterschiedlichen Bedürfnisse von Interessengruppen einzugehen. Eine weitere zentrale Forderung von VolksvertreterInnen ist, bei der Festsetzung von Tarifen und der Auswahl des Anbieters mitbestimmen zu können.

Angesichts knapper Finanzen werden lokale Nichtregierungsorganisationen (NGOs) zunehmend in Partnerschaften mit öffentlichen Institutionen und privaten Unternehmen eingebunden. Im Lager der NGOs ist man darüber geteilter Meinung. Die eine Fraktion sieht in der Kooperation eine Möglichkeit, ihre eigenen Interessen gegenüber dem Privatsektor zu vertreten und so Schäden im Rahmen von Privatisierungen abzufedern. Gegner der Privatisierungen lehnen jedoch die Zusammenarbeit mit der Privatwirtschaft und dem Staat vehement $\mathrm{ab}$, da für sie eine widersprüchliche Beziehung zwischen privatem Profit und den Verteilungsaufgaben des Staats besteht, die von privaten Unternehmen nicht übernommen werden kann und darf ${ }^{8}$.

NGOs im Norden und Süden, welche die Interessen der Armen anwaltschaftlich vertreten, warnen vor übertriebenen Hoffnungen und vor der Illusion, mit öffentlich-privaten Partnerschaften eine einfache, überall praktikable Lösung für die Bereitstellung von Basisinfrastruktur in Entwicklungsländern gefunden zu haben. Ihre Vorbehalte, auf die wir nachfolgend näher eingehen, stützen sich auf praktische Erfahrungen aus aller Welt.

6 R. Hemming und T. Ter-Minassian, ,Making Room for Public Investment“, Finance \& Development, IWF, Dezember 2004.

Ibid.

8 A. Escobar, „,The Making and Unmaking of the Third World through Development“, in M. Rahnema und V. Bawtree (dir.), The Post Development Reader, London, Zed Books, 1997, S. 85-93, zitiert in F. Miraftab, „Public-Private Partnerships, the Trojan Horse of Neoliberal Development ?“, op. cit., S. 90. 
Bevor wir die Diskussion über die öffentlich-privaten Partnerschaften vertiefen, wollen wir zunächst die Hintergründe zur Entstehung des Partnerschaftsmodells aufzeigen. PPPs sind unseres Erachtens das Resultat verschiedener entwicklungspolitischer Paradigmen der Politik der internationalen Finanzinstitutionen, welche die oben beschriebene Komplexität von Aus- und Wechselwirkungen nicht ausreichend untersuchten und diese unterschätzten.

Nach dem Zweiten Weltkrieg widmete sich die internationale Entwicklungspolitik vierzig Jahre lang der Stärkung von Regierungen in der Dritten Welt. Nach der Entkolonialisierung galt es vor allem, nationalistische Bestrebungen zur Bildung der neuen multi-ethnischen Staaten zu fördern. Dabei ist nicht zu vergessen, dass die internationale Gebergemeinschaft die Regierungen pro-westlicher Entwicklungsländer als Bollwerk gegen den Kommunismus stärkte. Die armen Länder sollten durch kreditfinanzierte Entwicklung den Anschluss an die Industrieländer schaffen. Die hohen und oftmals unproduktiv eingesetzten Kredite führten jedoch zu Beginn der 80er Jahre zum Ausbruch der weltweiten Schuldenkrise. Zu deren Bekämpfung propagierten der Internationale Währungsfonds (IWF) und die Weltbank ein neues entwicklungspolitisches Paradigma, den „Washington-Konsensus“. Dieser basiert auf der neoliberalen Politik von Ronald Reagan und Margaret Thatcher und propagiert, dass Märkte die Verteilung von Gütern besser regeln als Regierungen. Damit stand fortan weniger die Stärkung von Regierungen sondern von Märkten im Zentrum ihrer Politik. Die Doktrin bildete auch die Grundlage der Strukturanpassungsprogramme, die IWF und Weltbank den meisten Entwicklungsländern in den 80er und 90er Jahren zur Bekämpfung der Schuldenkrise auferlegten. Die Massnahmen zur Sanierung der Staatsfinanzen umfassten die Liberalisierung der Wirtschaft, die Öffnung von Märkten für ausländische Investitionen, die Privatisierung früherer staatlicher Betriebe, eine rigorose Sparpolitik des öffentlichen Sektors und die Förderung von Exportprodukten zur Erwirtschaftung von Devisen.

Die Folgen dieser Politik sind hinlänglich bekannt ${ }^{9}$. Einsparungen im Gesundheits- und Bildungswesen und der massive Abbau von Regierungsstellen trugen dazu bei, dass die Anzahl der „neuen Armen“ in den Grossstädten des Südens zunahm. Scharen von Beamten wurden entlassen, ohne dass neue Unternehmen bereit standen, um sie aufzufangen. Besonders viele Frauen, die im Staatssektor überdurchschnittlich vertreten sind, gerieten in ausweglose Situationen. So riefen die Anpassungsprogramme statt einer Lösung zur Reduzierung des Schuldenüberhangs neue strukturelle Probleme hervor. Es gab mehr Arbeitslose, die weniger investierten, somit weniger Nachfrage nach Konsumgütern und weniger Investitionen. Die nicht angekündigten Folgen dieser Spirale waren die Zunahme von Schattenwirtschaft und Schwarzarbeit, Korruption und Kriminalität sowie soziale Ungleichheiten. Viele Länder haben dadurch heute weniger

9 Siehe: Structural Adjustment Participatory Review International Network (SAPRIN), The Policy Roots of Economic Crisis and Poverty: A Multi-Country Participatory Assessment of Structural Adjustment, Washington, D.C., SAPRIN, 2002, (SAPRIN-Report, eine Analyse aus 50 Ländern über die Auswirkungen der Strukturanpassungspolitik), <www.saprin.org>. 
Geld für öffentliche Aufgaben und soziale Wohlfahrt, als vorher. Die Verkleinerung von staatlichen Institutionen reduzierte zudem die Handlungsautonomie der jeweiligen Regierungen und der nationalen Parlamente und schränkte den Spielraum für Aufgaben der öffentlichen Wohlfahrt massiv ein. Betroffene aus dem Süden werfen den Banken und privaten Unternehmen vor, ihr Handeln mehr an ihrem eigenen Gewinnstreben zu orientieren, als um das Schicksal der Armen besorgt zu sein.

Als Antwort auf die heftige Kritik an den Strukturanpassungen und deren Folgen propagierten IWF und Weltbank im Jahr 2000 einen neuen Paradigmenwechsel. Seitdem müssen arme Länder Armutsbekämpfungsstrategien mit der Bevölkerung erarbeiten und ihre öffentlichen Ausgaben danach ausrichten. Allerdings ist die Beteiligung des Privatsektors weiterhin Bestandteil von Kreditbedingungen. Nachdem sich Unternehmen des Nordens in den letzten zehn Jahren bereits die „Leckerbissen“ aus der Privatisierung der Staatsbetriebe in Ländern des Südens und Ostens gesichert haben, ist es heute schwieriger, ausländische Investoren zu gewinnen. Um ihnen Auslandsinvestitionen schmackhaft zu machen, heisst die Parole der Weltbank nun nicht mehr „Privatisierung“, sondern „Beteiligung des Privatsektors“ und „PPPs“. Die Schlagwörter „Beteiligung" und „Partnerschaft" sollen Unternehmen signalisieren, dass das Unternehmensrisiko im Notfall vom Staat getragen wird.

\section{Reform des Staatssektors als ernsthafte Option?}

NGOs, Bürgergruppen und Wissenschaftler fragen zunehmend lauter, weshalb Reformen des Staatssektors nicht ernsthafter angegangen werden und stattdessen internationale Geber und Finanzinstitutionen nach wie vor auf Privatisierung und PPPs setzen. Denn nach 25 Jahren Strukturanpassung weisen Regierungen in Entwicklungsländern immer noch grosse institutionelle Defizite auf ${ }^{10}$. Eine Erklärung dafür mag sein, dass sich in Entwicklungsländern häufig vor allem hohe Regierungsbeamte nicht ganz uneigennützig für Partnerschaften mit der Privatwirtschaft einsetzen. Manche werden selbst Manager der Unternehmen, oder sie vergeben Aufträge an Familienmitglieder.

Eine andere Ursache ist in der Strategie von IWF und Weltbank zu suchen. Zum einen war es diesen Institutionen wichtiger, dass Schuldnerländer die Devisen für die Bezahlung der internationalen Kreditforderungen erwirtschaften, weshalb fiskalische Bedingungen und Einsparungen im Vordergrund ihrer Politik standen. Zum anderen halten sie den Regierungssektor für kaum reformierbar. Die Weltbank argumentiert, dass Manager staatlicher Betriebe weniger effektiv arbeiteten, da sie immer damit rechnen könnten, öffentliche Zuschüsse zu erhalten. Ausserdem würden Regierungsbeamte nicht im öffentlichen Interesse handeln, sondern seien versucht, sich über ihre Position Vorteile zu verschaffen. Mangelnde Anreize und hohe Transaktionskosten zur Einführung von Überwachungssystemen würden Qualitäts- und Effizienzsteigerungen der Arbeit von

10 T. Mkandawire und C. Soludo, Our Continent, Our Future: African Perspectives on Structural Adjustment, Trenton (New Jersey), Africa World Press, 1999, S. 135 : „Die Notwendigkeit, die administrativen Strukturen afrikanischer Verwaltungen zu reformieren, um die Effizienz zu steigern, die öffentliche Rechenschaftspflicht zu erhöhen und die Korruption einzudämmen, ist nach wie vor offensichtlich". 
Beamten unmöglich machen. Nicht zuletzt wirke sich die private Konkurrenz langfristig auch positiv auf die Effizienz im öffentlichen Sektor aus ${ }^{11}$.

Der Druck der Zivilgesellschaft brachte die Weltbank und den IWF dazu, seit dem Jahr 2000 verstärkt Massnahmen zur guten Regierungsführung in Kreditbedingungen aufzunehmen. Doch die starke Einbeziehung des Privatsektors offenbart gleichzeitig den grossen Einfluss der Privatwirtschaft auf die Weltbank und die Ministerien der Entwicklungszusammenarbeit im Norden.

\section{Beurteilung des Partnerschaftskonzepts PPPs}

\section{Sind Regierungen überhaupt für Public-Private-Partnerships gerüstet?}

Die Theorie von öffentlich-privaten Partnerschaften, dass der Staat steuert und dabei die verschiedenen Interessen und Bedürfnisse des Privatsektors und der Zivilgesellschaft berücksichtigt, setzt politischen Willen und eine entsprechende staatliche Regulierungsfähigkeit voraus. Dazu müssten Regierungen die Rolle eines Mediators übernehmen, um die Gleichberechtigung der Partner zu garantieren. Dies bestätigen auch PPPs, bei denen die Auslagerung ehemals staatlicher Aufgaben zu einer einseitigen Bevorzugung ethnischer Gruppen oder ausländischer Konzerne führte und dabei erhebliche ethnische Spannungen und Proteste hervorrief. So hat beispielsweise die malaysische Regierung mittlerweile bestimmt, dass 30 Prozent der privatisierten Betriebe in den Besitz von Ureinwohnergruppen gehen müssen. Diese hatten sich in heftigen Protesten beklagt, dass chinesische Unternehmer bevorzugt würden ${ }^{12}$.

NGOs fragen sich angesichts der Nicht-Reformierbarkeit des Regierungssektors, ob staatliche Behörden überhaupt für Partnerschaften mit dem Privatsektor gerüstet sind. Viele Partnerschaftsprojekte in Südafrika zeigen zum Beispiel, dass insbesondere Angestellte lokaler Gemeinden den vielfältigen Aufgaben im Rahmen von Partnerschaften auf dezentraler Ebene nicht gewachsen sind ${ }^{13}$. Aber auch in den Zentralregierungen fehlt es den verkleinerten Ministerien oft an Personal, Kompetenzen und Willen zur Überwachung der Regulierungsbehörden und zur Ausgestaltung von Verträgen. Während den privaten Partnern gut bezahlte internationale Rechtsanwälte zur Seite stehen, herrscht diesbezüglich ein eklatantes Defizit auf Seiten der Regierungen in Entwicklungsländern ${ }^{14}$. Es fragt sich daher schon, wie dieselbe Regierung, die von Weltbank und IWF für nicht reformfähig befunden wurde, nun die fachlichen Kapazitäten für die Ausgestaltung und Überwachung komplexer PPP-Verträge aufbringen soll.

Das wirft die Frage auf, wer in den Partnerschaftsverträgen die Interessen der Nutzniesser, also der Zivilbevölkerung, vertritt. Man kann nicht grundsätzlich davon ausgehen, dass sich Regierungsbeamte hierzu verpflichtet fühlen, wie die

11 Y. Bangura, Public Sector Restructuring: The Institutional and Social Effects of Fiscal, Managerial and Capacity-Building Reforms, Occasional Paper, No 3, Geneva, United Nations Research Institute for Social Development (UNRISD), 2000, S. 5.

12 Y. Bangura, op. cit., S. 21 : Ähnliche Probleme gab es auch in Tansania und Nigeria.

13 F. Miraftab, op. cit., S. 94 : Diverse Beispiele aus Südafrika.

14 Siehe dazu: T. Kessler, „Putting the Private Sector in its Place“, Part 1, Issues and Evidence in the Reform of Water and Electricity Services; Citizens' Network on Essential Services, Takoma Park, USA, 2002, S. $10 \mathrm{ff}$. 
oben aufgeführten Argumente der Weltbank zeigen. Hinzu kommt, dass der im Norden historisch verankerte Gesellschaftsvertrag über die soziale Verantwortung der Regierung in Entwicklungsländern nicht ausgeprägt ist. Beim Bau von Basisinfrastruktur müssten Kooperationsverträge von vornherein nicht nur die Interessen und Verpflichtungen von staatlichen und privaten Partnern, sondern auch der Bevölkerung berücksichtigen. Gewöhnlich blenden Verträge jedoch Fragen über die Teilung der Verantwortung zwischen Staat und Privaten ebenso aus wie das politische, wirtschaftliche, soziale und kulturelle Umfeld und die Forderungen der betroffenen Bevölkerung ${ }^{15}$. Das mag daran liegen, dass Unternehmen befürchten, die Berücksichtigung aller Interessengruppen werde kompliziert, langwierig und dadurch teuer. Der betroffenen Bevölkerung bleibt oftmals nichts anderes übrig, als durch öffentliche Demonstrationen und Strassenblockaden die Regierung zu zwingen, auf ihre Bedürfnisse einzugehen. Das unten aufgeführte Beispiel der Wasserversorgung in El Alto, Bolivien, ist dafür exemplarisch. Die Proteste offenbaren ein weiteres, gravierendes Problem: Obwohl der Staat theoretisch die Kontrolle über die öffentlich-privaten Partnerschaften hat, werden besonders bei langfristigen Verträgen weite Bereiche der wirtschaftlichen Verantwortung für öffentliche Dienste an Private abgegeben und damit der demokratischen Kontrolle entzogen. Weder Aktionärsversammlungen, noch Parlamente können über die „Zwischenwelt“ der PPPs zur Rechenschaft gezogen werden.

Schliesslich sind Entwicklungsexperten zunehmend skeptisch, ob Regierungen wirklich durch öffentlich-private Partnerschaften finanziell profitieren. Die Privatisierungswelle der letzten Jahre zeigte, dass Regierungen nur geringfügig mehr Steuern einnahmen, weil viele der privaten Partner ausländische Firmen sind, die sich gerade aus Steuervorteilen auf das Joint-Venture einliessen. Selbst führende Mitarbeiter des IWF warnen mittlerweile vor übereilten öffentlich-privaten Partnerschaften, weil nicht nachgewiesen werden konnte, dass PPPs Korruption und Misswirtschaft beseitigen und zu Armutsbekämpfung führen ${ }^{16}$.

Das folgende Beispiel der Hausbaupolitik der südafrikanischen Regierung in den 90er Jahren verdeutlicht, dass Partnerschaften dann scheitern, wenn die Interessen und Bedürfnisse der Armen nicht berücksichtigt werden.

\section{Lehren aus dem Scheitern der südafrikanischen Public-Private-Partnership in der Hausbaupolitik ${ }^{17}$}

1994 rief die südafrikanische Regierung eine neue Hausbaupolitik ins Leben. Mit Hilfe eines Subventionsprogramms und auf der Basis einer Partnerschaft zwischen Armen, privaten Baufirmen und lokalen Regierungen sollten innerhalb von fünf Jahren eine Million Häuser für benachteiligte Familien gebaut werden. Die staatliche Unterstützung wurde jedoch nicht direkt an die neuen Hausbesitzer, sondern an neu gegründete Hausbaugenossenschaften ausbezahlt. Im Jahr 2000 war klar, dass nicht einmal die Hälfte der geplanten Häuser stand und somit die neue Politik gescheitert war. Was war falsch gelaufen?

15 U. Hoering, „Entwicklungspartnerschaften - Eine wunderbare Freundschaft?“, Weed-Arbeitspapier, Bonn, World Economy, Ecology and Development (WEED), 2003, S.10.

16 IWF, Finance \& Development, Dezember 2004, op. cit., S. 31.

17 F. Miraftab, op. cit., S. 95. 
Zum einen wurden Entscheidungen von Gemeinden und Banken über die Ausgestaltung der Hausbaupolitik ohne den Einbezug von Basisorganisationen und von den zukünftigen Hausbesitzern getroffen. Tatsächlich waren die geplanten Häuser sehr klein, von schlechter Qualität und lagen so weit ausserhalb von Stadtzentren und Arbeitsplätzen, dass viele Familien kein Interesse an einem teuren Umzug hatten. Zum anderen war die Hausbaupolitik von den Interessen marktwirtschaftlicher Institutionen wie den Banken und professionellen Baufirmen geprägt. In die Baugenossenschaften kauften sich nur private Firmen ein, welche die Häuserpreise allein festsetzten. Politiker gingen davon aus, dass der private Banksektor den armen Familien Kredite für den Hauskauf zur Verfügung stellen werde. Die südafrikanische Regierung beschränkte sich deshalb auf Massnahmen zur Risikoabdeckung der Privatbanken. Doch die Banken vergaben zur Minimierung ihres Risikos nur Kredite an Familien, die einigermassen finanzkräftig schienen. Dies schloss jedoch 70 Prozent der Zielgruppe von Bankkrediten aus. Damit scheiterte das gesamte Modell, denn ohne einen finanziellen Eigenanteil baute die Baugenossenschaften keine Häuser, und damit verfiel der Anspruch armer Familien auf staatliche Subventionen. Die südafrikanische Vereinigung von Wohnungslosen, die über hunderttausend Mitglieder zählt, beklagte sich bei der Regierung über das Vorgehen. Die „Partnerschaft für den Hausbau" hat jedoch bis heute keine Massnahmen getroffen, um arme Familien, zum Beispiel durch Finanzintermediäre und Spargruppen, mit den Banken zu verbinden und ihnen so den Zugang zu Bankkrediten und damit zu den Häusern zu sichern.

\section{Spannungsfelder zwischen privaten Anbietern und öffentlichen Aufgaben}

Wie wir bereits aufgezeigt haben, gibt es zahlreiche Spannungsfelder zwischen den Interessen privater Unternehmen und den Aufgaben von öffentlichen Institutionen, die Partnerschaften mitunter sehr schwierig machen. Die Literatur über die Vorteile von PPPs befasst sich jedoch kaum mit innovativen Lösungsansätzen zu folgenden Fragen: Wie kann zum Beispiel eine Brücke geschlagen werden zwischen den betriebswirtschaftlichen Rentabiliätsanforderungen privater Unternehmen und den entwicklungspolitischen Zielen von Regierungen? Sind unternehmerische Entscheidungsfreiheit und öffentliche Regulierungsanforderungen überhaupt partnerschaftlich vereinbar? Schliessen sich privates Gewinnstreben und öffentliche Versorgungsaufgaben für einkommensschwache Bevölkerungsgruppen nicht aus? Wie soll man umgehen mit der demokratischen Pflicht zur Offenlegung von Vertragsvereinbarungen im öffentlichen Interesse und ihrer Geheimhaltung aus Furcht vor Konkurrenten? Oder wie kann man dem Wunsch von Unternehmen nach schnellen Entscheidungen und dem Zeitaufwand, den eine Prüfung von Umwelt- und Sozialverträglichkeit erfordert, gerecht werden $?^{18}$. Wir wollen im Folgenden zumindest auf einige dieser Aspekte eingehen.

Öffentlich-private Partnerschaften im Bereich der Basisinfrastruktur sind häufig durch fehlende Konkurrenz gekennzeichnet, was die privaten Partner zu Monopolanbietern macht. Dadurch können sie die Preise konkurrenzlos anheben. Von Preissteigerungen für Basisdienste sind Menschen an der Armutsgrenze am

18 U. Hoering, 2003, op. cit., S. 12. 
stärksten betroffen. NGOs kritisieren, dass PPP-Verträge in den meisten Fällen die Unternehmen nicht dazu verpflichten, Aufgaben im Sinne der öffentlichen Wohlfahrt zu übernehmen. Solche Aufgaben wären zum Beispiel, auch arme Stadtteile an die Wasser- und Stromversorgung sowie die Abwasserentsorgung anzuschliessen oder die Preise der Zahlungskraft der Konsumenten anzupassen. Regierungen, Geber und internationale Finanzinstitutionen scheinen geradezu als „fait accompli“ hinzunehmen, dass die privaten Partner nur in den lukrativen Bereichen operieren. Oftmals können es sich die auf Investoren angewiesenen Regierungen nicht leisten, den privaten Partnern einschneidende Auflagen zu machen. Diese wiederum nutzen ihre monopolistische Stellung aus, um Gewinne zu erzielen, und rechtfertigen ihr Vorgehen mit ihrem hohen Risiko.

Eine Form von Partnerschaften im Infrastrukturbereich, in denen private Unternehmen als Monopolisten auftreten, sind so genannte „Build-Own-OperateTransfer" (BOOT)-Projekte. Seit sich Regierungen von Entwicklungsländern durch gigantische und zum Teil unproduktive Grossprojekte wie Staudämme, Strassen und Pipelines hoch verschuldet haben, rät die Weltbank bei grossen Infrastruktur-Bauvorhaben in Entwicklungsländern zu dieser Form von Partnerschaft. Private Investoren werden mit einer Investitionsgarantie der Regierung oder der Weltbank angelockt und sollen in unterschiedlichem Ausmass bislang öffentliche Aufgaben übernehmen. So bauen und betreiben sie zum Beispiel Elektrizitätswerke, Wasserkraftwerke oder Wasserleitungssysteme und wickeln die Versorgung der Kunden und die Rechnungen ab. Das Eigentum an den Einrichtungen sowie die Kontrolle über den privaten Betreiber bleiben hingegen staatlich. Im folgenden Beispiel zeigen wir anhand des geplanten Bujagali-Staudammprojekts in Uganda, welche Probleme BOOT-Projekte für die betroffene Bevölkerung aufwerfen können.

\section{Probleme durch B00T-Projekte am Beispiel des geplanten Bujagali- Staudamms in Uganda}

Seit zehn Jahren plant die ugandische Regierung in Bujagali am Weissen Nil, in der Nähe des Victoriasees, ein Wasserkraftwerk mit einer Leistung von 200 Megawatt zu bauen, um die Energieknappheit des Landes zu verringern. 40 Prozent der Bevölkerung Ugandas leben unterhalb der Armutsgrenze, und nur 3 Prozent haben Zugang zu Strom. Weil Uganda hoch verschuldet ist, kann sich die Regierung den Bau aus eigenen Mitteln nicht leisten. Im Rahmen eines BOOTVertrages vergab sie deshalb 1994 die Verantwortung für den Bau und die Betreibung an die US-amerikanische Baufirma Applied Energy Systems Nile Power (AESNP), mit der Auflage, das Kraftwerk dreissig Jahre lang zu betreiben. Den grössten Teil des 550 Millionen Dollar teuren Projekts sollten die Weltbank, die Afrikanische Entwicklungsbank sowie Exportkredite und Garantien aus der Schweiz, Schweden, Norwegen und Holland finanzieren ${ }^{19}$. Die schweizerische Niederlassung der Firma Alstom hatte einen Lieferauftrag über 50 Millionen Franken $^{20}$. 2003 platzte das Geschäft mit AES jedoch. Ausschlaggebend war,

19 Presseinformationen von AES-Nilepower, 20.12.2001, <http://www.aes.com/aes/index?page=news\& reqid $=239324>$.

20 Informationen von Alstom Schweiz sowie African Energy, No 75, Juni 2004, <http://www.africaenergy.com/html/public/data/ugandaAE.html>. 
dass die Firma AES sich bei anderen Projekten in Südamerika stark verschuldet hatte und ihr offenbar das Risiko zu gross war, den Bujagli-Staudamm zu bauen. Das Beispiel offenbart damit die Gefahren einer Auslagerung öffentlicher Dienstleistungen an private Akteure. Der Grossstaudamm sorgt zudem bei Umweltschützern für Kritik. Er würde ein bedeutendes Biotop an der Quelle des Nils und, mit den Bujagali-Wasserfällen, ein einmaliges Naturschauspiel sowie eine bedeutende Touristenattraktion überschwemmen. Zudem wurden bereits hundert Fischerfamilien umgesiedelt, die nun ohne Landrechte und ohne Strom, weit vom Fluss entfernt, auf einem Berg leben.

Der grösste Kritikpunkt von NGOs war der Inhalt eines zunächst geheimen Energieabnahmevertrags, der erst 2003 auf öffentliches Betreiben in einem richterlichen Beschluss veröffentlicht wurde. Er enthielt zahlreiche Klauseln, die international unüblich sind. Die ugandische Regierung musste sich verpflichten, ihre Elektrizitätsbehörde zu privatisieren und die nationalen Stromleitungen zu erneuern und auszubauen. Weiter hätte sie während dreissig Jahren den Strom von AESNP zu einem festen und im internationalen Vergleich sehr hohen Preis abkaufen müssen, unabhängig davon, ob überhaupt Strom produziert oder benötigt wird. Es stellte sich heraus, dass der Abnahmepreis so unverhältnismässig hoch war, weil AESNP überteuerte Bau- und Betreibungskosten angab. Der Vertrag begünstigte klar die Baufirma, und der überteuerte Stromabnahmepreis hätte sich in überhöhten Strompreisen für die Bevölkerung niedergeschlagen. Tatsächlich wäre der Strompreis etwa gleich teuer wie in der Schweiz gewesen, was sich nur die kleine reiche Oberschicht Ugandas hätte leisten können. Um AESNP den Strom abzunehmen, sah die ugandische Regierung vor, einen Teil des Stroms an Kenia und Tansania zu verkaufen, wobei mit beiden Regierungen noch keine Abnahmeverträge bestanden ${ }^{21}$. NGOs kritisierten insbesondere auch die Weltbank, die sich nicht für eine gerechte Risikoverteilung zwischen den Parteien und für den Einbezug der Interessen der armen Bevölkerung einsetzte.

Die Erfahrungen des Bujagali-Projekts zeigen, dass bei BOOT-Projekten eine Veröffentlichung der Energieabnahmeverträge im Sinne demokratischer Mitsprache zwingend sein muss, damit die Bevölkerung nicht übervorteilt wird. Es wird auch offensichtlich, dass weder die Regierung, noch der private Partner ein Interesse an den Nöten und Bedürfnissen der armen Bevölkerung haben: Das vorsätzliche Interesse des privaten Unternehmens ist, das Risiko zu mindern und Gewinne zu erzielen. Die Vertreter der Regierung wollen mit dem Grossstaudamm ein nationales Prestigeprojekt verwirklichen, mit dem sie im Zeichen wirtschaftlicher Entwicklung jedoch in erster Linie ihren persönlichen Namen und politische Einflussnahme verknüpfen. Auch die Rolle der Weltbank bleibt undurchsichtig. Auf jeden Fall löste sie ihren Anspruch nicht ein, dass durch ihre Beteiligung Projekte sozialer und ökologisch verantwortlicher würden. Denn nach dem Scheitern des Projekts endete auch der Vertrag mit der Weltbank, ohne dass sich jemand um die bereits umgesiedelten Fischerfamilien und deren Schicksal kümmerte.

Im April 2005 hat die ugandische Regierung nun mit der Agha-Khan-Stiftung einen neuen Vertrag über den Bau des Staudamms unterzeichnet. Das gleiche

21 Prayas, Energy Group, Pune, India, „The Bujagali Power Purchase Agreement: an Independent Review“, Berkeley, California, International Rivers Network, November 2002, <http://www.irn.org/ programs/bujagali/bujagalippa-review.pdf $>$. 
Projektdesign wie vorher soll nun für 350 Millionen Dollar (statt 550 Millionen) gebaut werden. Lokale Parlamentarier kritisieren, dass selbst die Redimensionierung für Uganda zu teuer sei und sich in zu hohen Stromtarifen niederschlagen werde. Zudem warnen Wasserexperten, NGOs und sogar Hydrologen des ugandischen Wasserministeriums, dass sich die stark schwankenden Wassermengen des Nils am geplanten Ort nicht für den Bau eines Staudamms eignen ${ }^{22}$. Die ugandische Regierung hält aus Prestigegründen weiter an ihren Plänen fest. Damit das Projekt dereinst gebaut wird, muss sie jedoch erneut internationale Finanzgeber überzeugen.

\section{Anschubfinanzierung durch Partner der Entwicklungszusammenarbeit}

Wie die obigen Beispiele zeigen, ist es im Bereich der Basisinfrastruktur kaum möglich, das Gewinnstreben des Privatsektors mit den öffentlichen Aufgaben der Armutsbekämpfung und der Entwicklung zu verbinden. Die gängige Kritik: „Die Gewinne privat, die Kosten dem Staat“, ist nicht von der Hand zu weisen. Je mehr ein privater Anbieter den Interessen der Ärmsten dienen soll, desto unattraktiver wird das Geschäft. Welches Interesse sollten Firmen haben, in Gegenden zu investieren, in denen die Mehrheit der Menschen unter der Armutsgrenze von weniger als einem Dollar pro Tag lebt? In solchen Fällen bleibt praktisch oft keine andere Möglichkeit als eine Subvention durch die Regierung, die Weltbank oder Partner der internationalen Entwicklungszusammenarbeit, welche in Gegenden, in denen viele arme Menschen leben, die hohen Kosten für Anschlüsse oder für den Bau von Leitungen übernehmen. Staatliche Subventionen haben den Nachteil, dass der private Anbieter damit „pokern“ kann, dass der Staat notfalls einspringt, und das Unternehmen die Anzahl der armen Haushalte ignorieren kann. Erfahrungen im Bereich der Wasserversorgung bestätigen, dass auch dieses Modell ein Fass ohne Boden ist. In Manila (Philippinen) und in Cochabamba (Bolivien) konnte sich die arme Bevölkerung das Wasser trotz staatlicher Subventionierung der Anschlüsse nicht mehr leisten, nachdem der private Anbieter die Wasserpreise um das Dreifache angehoben hatte ${ }^{23}$. Die Regulierungsbehörden waren zu schwach, um die Monopolisten zu kontrollieren. Weitere Nachteile von Subventionen sind die hohen administrativen Kosten für die Regierung. Die Einsparungen durch die Auslagerung von Dienstleistungen an private Partner werden dadurch obsolet.

Zudem wirkt sich nun nachteilig aus, dass Regierungen weniger Einnahmen haben, wenn private Unternehmen die Versorgung von wohlhabenden Gegenden übernehmen. Dadurch sind kaum mehr öffentliche Quersubventionen möglich. In diese Lücke springen zunehmend internationale und nationale Entwicklungsorganisationen. Auch das Staatssekretariat für Wirtschaft (seco) versucht im Rahmen seines PPP-Programms, eine solche Art von Anschubfinanzierung zu leisten. Das seco hat jedoch noch keine nachhaltigen Erfahrungen mit dem Instrument. Das erste Projekt dieser Art, die Subventionierung

22 Charles Ariko, „Dam Bid Attacked Again“, The New Vision Newspaper, Uganda, 19.4.2005, sowie: National Association of Professional Environmentalists (NAPE), Kampala, „Uganda Civil Society Workshop regarding Bujagali Power Project“, Hotel Equatoria, Kampala, Uganda, 15.4.2005.

23 D. Hall, „The Water Multinationals 2002: Financial and Other Problems“, London, Public Services International Research Unit (PSIRU), 2002. 
der Wasseranschlüsse im armen Teil von La Paz (Bolivien) mit Schweizer Steuergeldern, ist im Januar 2005 gescheitert. Darauf gehen wir nachfolgend näher ein.

\section{Probleme mit öffentlich-privaten Partnerschaften am Beispiel der Wasserversorgung in El Alto, Bolivien}

\section{Das seco-Projekt zur Wasserversorgung und Abwasserentsorgung in El Alto}

Die Stadt El Alto liegt auf 4000 Meter über dem Meer auf dem trockenen Altiplano, oberhalb der Haupstadt Boliviens, La Paz. 700'000 Menschen leben hier in einem wirren Geflecht von Lehmhütten und staubigen Strassen. 73 Prozent der Einwohner, meist Indios, die ihr Land verliessen, haben nur ein paar Dollar am Tag zum Überleben. In den meisten Quartieren gibt es keine Trinkwasserund Abwassersysteme. 1997 beschloss die bolivianische Regierung, diesen Missstand mit Hilfe von privaten Partnern in den Griff zu bekommen. Bolivien galt damals als der Musterschüler von IWF und Weltbank, der die Auflagen zur Privatisierung wie vorgegeben umsetzte. Den Konzessionsvertrag für die Wasserversorgung von La Paz und El Alto gewann die Firma Aguas del Illimani $(\mathrm{AdI})^{24}$, ein Konsortium unter der Leitung der französischen Grosskonzerns Suez Lyonnaise des Eaux. Sie verpflichtete sich für dreissig Jahre, 50 Prozent der Neuanschlüsse in Gebieten ohne existierende Hauptleitungen zu erstellen. Die Interamerikanische Entwicklungsbank und die Weltbank stellten Kredite von 35 Millionen Dollar in Aussicht. AdI rechnete mit jährlichen Betriebsausgaben von 20 Millionen Dollar. Über den erwarteten Gewinn wurden hingegen keine Zahlen veröffentlicht. In den ersten fünf Jahren erfüllte AdI die Auflagen und schloss 75'00 Haushalte an die Trinkwasserversorgung und 55'000 an die Abwasserentsorgung an.

Einer der am schnellsten wachsenden Stadtbezirke ist der „Distrikt 7“ mit 25’000 Einwohnern. Hier steigt die Gesundheits- und Epidemiegefahr stetig, weil sanitäre Anlagen oder Latrinen fehlen und die untiefen Brunnen oft mit Kolibakterien verseucht sind. Schon lange fordert die Bevölkerung, an die Wasserversorgung angeschlossen zu werden. Doch für den Konzern ist der Distrikt nicht rentabel, und die Stadt El Alto hat keine Gelder für den Bau der Wasser- und Abwasserleitungen. Die Zahlungskraft der Bevölkerung ist zu schwach, um die Kosten eines Neuanschlusses zu decken, welche die Regulationsbehörde in den letzten Jahren mehrfach angehoben hat. Ein Anschluss kostet momentan 445 US-Dollar und macht die Hälfte des Jahreseinkommens einer armen Familie aus. Zudem sind die Anschlussgebühren an den US-Dollar gebunden, was bei einer allfälligen Verteuerung des Dollars zu einem Tarifanstieg führen würde.

Das seco beschloss 2003, den Distrikt 7 durch einen nichtrückzahlbaren Zuschuss von 3 Millionen Dollar zu unterstützen. Damit sollten die Hauptleitungen und die Abwasserreinigungsanlagen finanziert und die Anschlussgebühren der armen Haushalte vorfinanziert werden. Durch Eigenleistungen der Bevölkerung sollten die Anschlusskosten auf 317 US-Dollar verringert werden. Zu deren

24 An AdI sind neben dem Hauptaktionär Ondeo-Suez (54\% Aktienanteile) die bolivianische Investment Corporation (22\%), die International Finance Corporation der Weltbank (8\%) und die Angestellten (2\%) beteiligt. 
Bezahlung hätte die Bevölkerung für fünf Jahre zinslose Darlehen beziehen können. Die Einnahmen wären in einen Rotationsfonds geflossen, um mit den Geldern später weitere Neuanschlüsse zu finanzieren. In einer ersten Phase sollten rund 3000 Trinkwasser- und 5000 Abwasseranschlüsse gebaut werden und in einer zweiten Phase nochmals 8500. Ingesamt hätten rund 60'000 Personen profitiert. Das seco legte Wert darauf, den Bedürfnissen der Betroffenen gerecht zu werden, und konsultierte die betroffene Bevölkerung. Auf deren Wunsch wurde in das Projekt auch ein Weiterbildungsprogramm in den Bereichen Bauarbeiten, Sanitärarbeiten, Hygieneerziehung und Stärkung von Quartiervereinen eingeplant. Ein Entscheidungsgremium (Comité Directivo) mit Beizug von VolksvertreterInnen sollte für Transparenz und für die richtige Verwendung der Gelder sorgen. Das seco hoffte, mit diesem Projekt zu demonstrieren, dass durch Partnerschaften auch in armen städtischen Randgebieten eine effiziente Wasserversorgung möglich ist. Das Modell sollte nach der Testphase in El Alto auf andere Länder und Infrastruktursektoren übertragbar werden ${ }^{25}$.

Doch soweit kam es bis jetzt nicht. Am 10. Januar 2005 gingen Tausende der Bewohnern El Altos auf die Strasse und forderten in tagelangen Demonstrationen die Verstaatlichung der privaten Wasserversorgung. Die Weltbank und die deutsche Kreditanstalt für Wiederaufbau (KfW) boten schliesslich zur Schlichtung der Krise und zur Rettung der Wasserversorgung Kredite in Höhe von 40 Millionen Dollar an, um die Anschlusskosten zu verringern. Vergebens, denn die Bevölkerung El Altos setzte sich durch. Am 13. Januar kündigte Boliviens Präsident Carlos Mesa den Konzessionsvertrag mit Suez Lyonnaise des Eaux, bzw. Aguas del Illimani. Damit platzte auch der Vertrag mit dem seco. Sofort wurde der Schwarze Peter hin und her geschoben: Die Volksvertreter aus den Armenvierteln in El Alto beschuldigten Suez Lyonnaise, sie nach sieben Jahren immer noch nicht an die Wasserversorgung angeschlossen zu haben. Die bolivianische Regulierungsbehörde war überrascht, dass der Präsident ohne rechtliche Grundlage den Konzessionsvertrag auflöste, und beschuldigte internationale NGOs, die Demonstranten von El Alto bezahlt zu haben. Der Chef der Regulationsbehörde sowie sein Stellvertreter traten von ihren Ämtern zurück, weil sie sich keiner Schuld bewusst waren. AdI sei auf ihre Empfehlungen zur Ausweitung des Konzessionsvertrags auf arme Gebiete nicht eingetreten. Der zuständiger Manager bei Suez Lyonnaise, Alain Mathys, schob die Schuld jedoch zurück: Er habe öfters darauf hingewiesen, dass es ungerecht sei, wenn eine Villa im Nobelvorort von La Paz gleich hohe Anschlussgebühren bezahle wie eine sehr arme Familie in El Alto. Aber die Regierung habe diese Politik so verabschiedet. Suez Lyonnaise sei durchaus bereit, die Preisstrukturen zu überprüfen, um gerechtere Tarife und Subventionsmöglichkeiten für arme Gebiete zu finden. Zur Überwindung der Krise sei der Konzern auch offen für eine staatlich-private Geschäftsführung von Aguas del Illimani ${ }^{26}$. Die Regierung sucht derweil fieberhaft nach einer Lösung. Sie hat mit der Auflösung des Konzessionsvertrags bereits 30 Millionen Dollar Schulden und ist mit verschiedenen Klagen lokaler Bauunternehmer und Lieferanten konfrontiert.

25 Projektunterlagen sowie mündliche Informationen des seco, Abteilung Infrastruktur, Februar 2005.

26 Juan Forero, „Latin America Fails to Deliver on Basic Needs“, New York Times, 22.2.2005, „Bolivian Government Signals Contract End“, Water 21 Magazin, Februar 2005, <http://www.iwahq.org.uk/ template.cfm ?name=bolivian_government_signals_contract_end $>$. 
Der Ruf der Bevölkerung El Altos nach einer Verstaatlichung der Wasserversorgung mag Aussenstehende auf den ersten Blick verblüffen, weil Bolivien in den 90er Jahren das Land mit der schlechtesten öffentlichen Wasserversorgung und Abwasserentsorgung ganz Lateinamerikas war. Die Gemeindeverwaltung von La Paz/El Alto galt nicht nur in Weltbankkreisen als korrupt und ineffizient ${ }^{27}$. Wenn die Bevölkerung nun lautstark die Rückkehr zur staatlich gemanagten Wasserversorgung fordert, geht es um viel Grundsätzlicheres, wie auch die Unruhen im Herbst 2003 zeigten, die zur Absetzung des Staatspräsidenten führten. Die Ablehnung der öffentlich-privaten Partnerschaft muss im Kontext der jahrhundertlangen Ausbeutung und Unterdrückung der Ureinwohner durch die Kolonialmächte gesehen werden. Diese findet nach Ansicht der Bevölkerung heute in der Ausbeutung und im Ausverkauf der natürlichen Ressourcen Boliviens durch transnationale Konzerne ihre Fortsetzung. Auch die im Geheimen geführten Verhandlungen zwischen der Regierung und den internationalen Finanzinstitutionen untermauern das Gefühl, von aussen bestimmt zu werden. Die Schuldzuweisungen über das Scheitern des Projekts zeigen zudem: Die bolivianische Regierung konnte der Bevölkerung nicht beweisen, dass sie die Kontrolle über die Partnerschaft mit dem Konzern Suez Lyonnaise des Eaux hat, weil die Kommunikation zwischen den Interessengruppen nicht funktionierte. Mögen die Anstrengungen des seco auch entwicklungspolitisch korrekt gewesen sein, sie bringen nichts, wenn das Projekt nicht von vornherein von der Mehrheit der Nutzniesser unterstützt wird.

Doch welche Möglichkeiten hat ein armes Land wie Bolivien, die Wasserversorgung in armen Gebieten zu gewährleisten? Eine Option ist die Privatisierung und die PPP-Strategie der Weltbank, die nun klar am Veto der Bevölkerung gescheitert ist. Eine zweite Möglichkeit sind Quersubventionen: Reichere Einwohner bezahlen höhere Tarife, um die Anschlüsse in ärmeren Gebieten zu unterstützen. Dies wäre jedoch nur mit einer starken, klassenunabhängigen und nicht-korrupten Regierung durchführbar, weil die Regierung selbst zur Kasse gebeten würde. Danach sieht es kaum aus, denn die reiche Oberschicht Boliviens ist ohne Druck durch die Regierung nicht bereit, sich für eine gerechte Verteilung im eigenen Land einzusetzen. Deshalb ist auch die dritte Option höherer Steuern wenig realistisch. Es gibt nur eine sehr kleine Mittelklasse, die Steuern bezahlt. Eine vierte Option, die NGOs weltweit fordern, ist die langfristige Stärkung der staatlichen Wasserversorgungsbehörden und des Regulators. Die internationalen Finanzinstitutionen und die bilateralen Partner der Entwicklungszusammenarbeit könnten über Kredite und Zuschüsse zur Budgethilfe Massnahmen zur Verbesserung der notwendigen Führungs- und Managementkapazitäten einfordern. Zudem muss ein Know-How-Transfer von den privaten Unternehmen zu den öffentlichen Akteuren durch entsprechende vertragliche Vereinbarungen eingeleitet werden.

Die Auflösung der Konzessionsverträge in La Paz bietet eine Chance für einen Neuanfang im Sinne einer demokratisch pluralistisch verstandenen Partnerschaft. Dazu sollte ein Multi-Stakeholder-Dialog einberufen werden, in welchem alle Interessenvertreter offen über Risiken, Gefahren und Chancen disku-

27 Washington Post, 10.2.2005. 
tieren und über Optionen verhandeln, wie sich auch Menschen in armen Quartieren Wasser leisten können.

Vorbild könnte die Wasserversorgung der bolivianischen Stadt Santa Cruz sein, die bereits 1979 ihre städtische Wasserversorgung einer Kooperative übergab. Die Kooperative SAGUAPAC funktioniert seitdem ebenso effizient wie ein privates Unternehmen und gilt als eine der am besten verwalteten Wasserwerke Lateinamerikas. Ihr Ziel ist nicht ausschliesslich, Gewinn zu erwirtschaften, sondern die Qualität der Wasserversorgung und die flächendeckende Versorgung der Bevölkerung zu verbessern.

\section{Die Erfolgsgeschichte der Kooperative SAGUAPAC in Santa Cruz, Bolivien²8}

Santa Cruz liegt im östlichen Tiefland Boliviens. Die isolierte Lage der Stadt förderte die regionale Identität und Autonomie. Seit in den 50er Jahren Öl- und Gasvorkommen gefunden wurden, wuchs Santa Cruz von 42'000 Einwohnern zu einer der wichtigsten Wirtschaftszentren Boliviens, mit heute über 1 Million Einwohnern. Trotz der hohen Zuwanderung etablierten sich in den 70er Jahren verschiedene soziale Vereinigungen und eine Bürgerbewegung zur Regelung öffentlicher Aufgaben im Geiste sozialer Gerechtigkeit. Aus dieser Bewegung heraus, und auch aus Abneigung gegen den schwachen und korrupten Regierungssektor und die oligarchisch strukturierte Privatwirtschaft, wurde 1979 die Wasserkooperative SAGUAPAC gegründet. Seither obliegt ihr allein die Verantwortung für die städtische Wasserversorgung.

Studien der Weltbank ${ }^{29}$ und der Universität Birmingham in England ${ }^{30}$ bezeichnen SAGUAPAC als eine der am besten verwalteten Wasserfirmen Lateinamerikas. Die Administrationskosten sind niedrig und die Zahl der Angestellten ist sehr gering. Auch der Wasserverlust ist sehr niedrig. Rund 96 Prozent der Kunden zahlen ihre Wasserrechnungen pünktlich. Trotz des grossen Bevölkerungswachstums sind 90 Prozent der Haushalte an die öffentliche Versorgung angeschlossen und erhalten 24 Stunden pro Tag sauberes Trinkwasser. Die Preise von SAGUAPAC basieren auf Quersubventionen: Jeder Haushalt muss einen geringen Basispreis bezahlen. Die weiteren Verbrauchertarife bemessen sich nach dem Einkommen. So liegen die Preise für kommerzielle Kunden bei 45 Prozent über denen für Privatpersonen. Haushalte am Existenzminimum bezahlen dagegen nur den Basistarif.

Warum funktioniert SAGUAPAC so erfolgreich? Die Kunden sind automatisch Mitglieder der Kooperative. Die Stadt ist in neun Wasserbezirke aufgeteilt, in denen die Kunden die Vertreter des Verwaltungsrats von SAGUAPAC ernennen. Dieser wiederum ernennt das Management und genehmigt die Wassertarife. Die Mitglieder ernennen zudem ein separates Gremium, welches den Verwaltungsrat

28 Siehe: SAGUAPAC, „Una Cooperativa en la Administración del Servicio de Agua y Alcantarillado“, <http://wbln0018.worldbank.org/LAC/lacinfoclient.nsf/4145fb3d8bc4c82c8525673900539662/ cb337cb6f3abd80785256cf000064d1a/\$FILE/SAGUAPAC.pdf>.

29 Worldbank, Operations Evaluations Department (OED), No 222, Bolivia Water Management: A Tale of Three Cities, 2002, S. 2.

30 A. Nickson, Organisational Structure and Performance in Urban Water Supply: The Case of the Saguapac Co-operative in Santa Cruz, Bolivia, IDD Working Papers, No 38, Birmingham, International Development Department (IDD), University of Birmingham, 2000. 
überwacht und dadurch Stabilität garantiert. Der Verwaltungsrat und die Direktoren sind seit Jahren engagiert. Sie rühmen die transparenten Strukturen, welche Korruption unterbinden und politische Einmischungen verhindern. Zudem gibt es keine Verzögerungen bei Ausschreibungen für Bauarbeiten, wie sie zum Beispiel in staatlichen Wasserversorgungsgesellschaften durch juristische Abklärungen und die Verwaltung von Krediten die Regel sind. Dadurch können Investitionen und Anschlüsse wesentlich schneller durchgeführt und Reklamationen effizienter behoben werden. Ein Schatten auf dem schönen Bild ist, dass verschiedene Mitglieder der Geschäftsführung seit einigen Jahren versuchen, neu zugewanderte Indio-Migranten zu diskriminieren. Sie versuchen, diese von der Kooperative auszuschliessen, um angeblich weitere Zuwanderungswellen zu verhindern.

Zwiespältig ist auch die Rolle der Weltbank. SAGUAPAC erhielt in den 90er Jahren zwei Kredite der Weltbank, die pünktlich zurückbezahlt wurden. Obwohl die Bank die effiziente Wasserversorgung der Kooperative lobte, propagierte sie das Modell nicht in anderen Städten. Stattdessen war die bolivianische Regierung 1998 von der Weltbank angehalten worden, bestehende Wasserkooperativen in Unternehmen mit privater Beteiligung umzuwandeln, um Investitionen privater Unternehmen zu fördern. Aus der Sicht von NGOs zeigt das Beispiel einmal mehr den massiven Einfluss internationaler Wasserkonzerne auf die Weltbank.

\section{Schlussfolgerungen und Empfehlungen}

Wir haben gezeigt, dass öffentlich-private Partnerschaften nur scheinbar eine Lösung zur Bereitstellung der Basisinfrastruktur für arme Bevölkerungsschichten bieten. Tatsächlich belegen die verschiedenen aufgeführten Beispiele von PPPs (Stromversorgung in Uganda, Wasserversorgung in La Paz (Bolivien) und Wohnungsbauprojekt in Südafrika), dass PPPs im Basisinfrastrukturbereich nicht funktionieren, wenn Regierungen zu wenig demokratisch ausgerichtet sind und ihren Auftrag der Armutsbekämpfung und der gerechten Verteilung öffentlicher Güter nicht ernst genug nehmen. Die Voraussetzungen für funktionierende PPPs sind jedoch in den wenigsten Entwicklungsländern gegeben, denn in vielen Ländern im Süden sind die Gerichte nicht unabhängig und Regulationsbehörden sowie Aufsichtsbehörden zu schwach, um die notwendige Kontrolle der PPPs zu gewährleisten. Unternehmen vermeiden nach Möglichkeit ein Engagement in unprofitablen Sektoren. Konkurrenz gibt es oftmals keine, weil die Märkte in ärmeren Ländern im Bereich der Basisinfrastruktur nicht funktionieren. Deshalb bleibt Regierungen meistens nichts anderes übrig, als wiederum die Versorgung armer Bevölkerungsschichten zu übernehmen und diese mit Steuermitteln, Krediten oder Zuschüssen externer Partner der Entwicklungszusammenarbeit zu finanzieren. Fragwürdig ist auch, wenn private Firmen direkt oder indirekt staatliche Subventionen erhalten, um die öffentliche Versorgung in Randgebieten zu sichern. Auch die Finanzabteilung des Internationalen Währungsfonds kritisiert an PPPs, dass Regierungen trotz der Auslagerung an private Investoren weiterhin das gesamte Risiko und noch dazu hohe administrative und soziale Kosten tragen, die schlussendlich durch die Steuerzahler aufgebracht werden ${ }^{31}$.

31 IWF, Finance \& Development, op. cit., S. 31. 
Wir warnen deshalb davor, im Bereich der Bereitstellung von Basisinfrastruktur öffentlich-private Partnerschaften als die beste Lösung zu propagieren. Dagegen fordern wir, dass staatliche Verteilungs-, Versorgungs- und Finanzierungsaufgaben in offenen, transparenten und partizipativen Prozessen geplant und durchgeführt werden, in denen die betroffene Bevölkerung von Anfang an beteiligt ist. Das Beispiel der bolivianischen Kooperative SAGUAPAC zeigt, dass ein gesamtgesellschaftlicher Dialog über alternative Entwicklungsmodelle und die Mitsprache der Kunden sehr effizient, gerecht, kostengünstig und nachhaltig sein kann.

Es ist an der Zeit, dass die internationale Gemeinschaft, wie Regierungen, Geber, die internationalen Finanzinstitutionen und die Privatwirtschaft eine Bestandsaufnahme über öffentlich-private Partnerschaften machen. Ihr Ergebnis sollte sein, die Lehren aus weltweiten Erfahrungen mit PPPs zu analysieren und zum einen eine klare Bestimmung der Bereiche vorzunehmen, in denen sowohl die Zielsetzung als auch die Finanzierung und Verwaltung öffentlich bleiben sollten. Zum anderen wäre eine weltweite Internetplattform sinnvoll, auf der gescheiterte und erfolgreiche PPPs sowie Alternativen aufgeführt werden. Daraus sollten international akzeptierte Standards resultieren, die der Öffentlichkeit, Regierungen und Gebern Anhaltspunkte liefern, ob und wann sie sich auf das Risiko einer öffentlich-privaten Partnerschaft einlassen können. 


\section{Quellen}

AES-Nilepower, Presseinformationen vom 20.12.200, <http://www.aes.com/aes/index?page=news \& reqid $=239324>$.

Bangura, Y., Public Sector Restructuring: The Institutional and Social Effects of Fiscal, Managerial and Capacity-Building Reforms, Occasional Paper, No 3, Geneva, United Nations Research Institute for Social Development (UNRISD), 2000.

Escobar, A., „The Making and Unmaking of the Third World through Development“, in M. Rahnema und V. Bawtree (dir.), The Post Development Reader, London, Zed Books, 1997, zitiert in F. Miraftab, „Public-Private Partnerships, the Trojan Horse of Neoliberal Development ?“, 2004, Journal of Planning Education and Research.

Forero Juan, „Latin America Fails to Deliver on Basic Needs“, New York Times, 22.2.2005.

Hall, D., „The Water Multinationals 2002 : Financial and Other Problems“, London, Public Services International Research Unit (PSIRU), 2002.

Hemming, R., Ter-Minassia, T., „Making Room for Public Investment“, Finance \& Development, International Monetary Fund, Vol. 41, No 4, December 2004.

Hoering, U., 2003, „Entwicklungspartnerschaften - Eine wunderbare Freundschaft?“, WeedArbeitspapier, Bonn, World Economy, Ecology and Development (WEED), 2003.

Kessler T., „Putting the Private Sector in its Place“, Part 1, Issues and Evidence in the Reform of Water and Electricity Services; Citizens' Network on Essential Services, Takoma Park, USA, 2002.

Miraftab, F, „Public-Private Partnerships, the Trojan Horse of Neoliberal Development ?“, Journal of Planning Education and Research, 2004.

Mkandawire T. und Soludo C., Our Continent, Our Future: African Perspectives on Structural Adjustment, Trenton (New Jersey), Africa World Press, 1999.

National Association of Professional Environmentalists (NAPE), Kampala, „Uganda Civil Society Workshop regarding Bujagali Power Project“, Hotel Equatoria, Kampala, Uganda, 15.4.2005.

Nickson, A., Organisational Structure and Performance in Urban Water Supply: The Case of the SAGUAPAC Co-operative in Santa Cruz, Bolivia, IDD Working Papers, No 38, Birmingham, International Development Department (IDD), University of Birmingham, 2000.

Osbourne S., Gabler T., Reinventing Government: How the Entrepreneurial Spirit is Transforming the Public Sector, Reading (Massachusetts), Addison-Wesley Pub. Co., 1992.

Prayas Energy Group, Pune, India, „The Bujagali Power Purchase Agreement: An Independent Review“, Berkeley, California, International Rivers Network, November 2002, <http://www. irn.org/programs/ bujagali/bujagalippa-review.pdf>.

Structural Adjustment Participatory Review International Network (SAPRIN), The Policy Roots of Economic Crisis and Poverty: A Multi-Country Participatory Assessment of Structural Adjustment, Washington, D.C., SAPRIN, 2002, <www.saprin.org>.

UN-Millennium Project, Jeffrey D. Sachs: „Investing in Development: A Practical Plan to Achieve the Millennium Development Goals“, United Nations Development Programme (UNDP), January 2005, Chap. 1.

Washington Post, USA, 10.2.2005.

Water 21 Magazine, Februar 2005, <http://www.environmental-expert.com/magazine/iwa/w21/>.

World Bank, World Development Report 1994, Infrastructure for Development, Washington, D.C.

World Bank, Operations Evaluations Department (OED), 2002, Number 222, Bolivia Water Management: A Tale of Three Cities. 